\title{
On the origin of the helium-rich population in the peculiar globular cluster Omega Centauri
}

\author{
Donatella Romano ${ }^{1,2}$, M. Tosi ${ }^{2}$, M. Cignoni ${ }^{1,2}$, F. Matteucci ${ }^{3}$, \\ E. Pancino ${ }^{2}$ and M. Bellazzini ${ }^{2}$ \\ ${ }^{1}$ Dept. of Astronomy, Bologna University, \\ Via Ranzani 1, I-40127, Bologna, Italy \\ email: donatella.romano@oabo.inaf.it \\ ${ }^{2}$ INAF-Bologna Observatory, \\ Via Ranzani 1, I-40127, Bologna, Italy \\ ${ }^{3}$ Dept. of Physics, Trieste University, \\ Via Tiepolo 11, I-34143, Trieste, Italy
}

\begin{abstract}
In this contribution we discuss the origin of the extreme helium-rich stars which inhabit the blue main sequence (bMS) of the Galactic globular cluster Omega Centauri. In a scenario where the cluster is the surviving remnant of a dwarf galaxy ingested by the Milky Way many Gyr ago, the peculiar chemical composition of the bMS stars can be naturally explained by considering the effects of strong differential galactic winds, which develop owing to multiple supernova explosions in a shallow potential well.
\end{abstract}

Keywords. globular clusters: individual (Omega Centauri) - galaxies: dwarf, evolution - stars: abundances, chemically peculiar

\section{A dwarf galaxy progenitor suffering strong galactic winds}

It has been suggested - and it is now widely accepted - that the kinematical, dynamical and chemical properties of the most massive globular cluster of the Milky Way, Omega Centauri ( $\omega$ Cen, NGC 5139), can all be understood if it is the surviving remnant of a larger system, captured and partially disrupted by the Milky Way many Gyrs ago (see, e.g., Romano et al. 2007, and references therein). However, the origin of the extreme He-rich stars hosted on its blue main sequence (bMS) still awaits a satisfactory explanation. Here we propose a possible solution, in the framework of a chemical evolution model which reproduces other major observed properties of the cluster, namely, its stellar metallicity distribution function (MDF), age-metallicity relation (AMR), trends of several abundance ratios with metallicity and $\mathrm{Na}-\mathrm{O}$ anticorrelation (Fig. 1; see Romano et al. 2007, 2010).

In order to reproduce all the relevant observations, the parent galaxy must experience both infall of gas of primordial chemical composition and outflow of processed matter. The key assumption that allows the formation of extreme He-rich stars is that, while supernova (SN) ejecta are efficiently lost through the galactic outflow, elements restored to the interstellar medium (ISM) by gentle winds from both asymptotic giant branch (AGB) and fast rotating massive stars (FRMSs) are mostly retained in the cluster potential well (see Romano et al. 2010 and references therein for details).

$\mathrm{He}$ and $\mathrm{Na}$ are dispersed in the ISM through slow stellar winds by both AGBs and FRMSs. O, instead, as well as other heavy elements, is expelled by massive stars through fast polar winds. Hence, in the framework of our model, $\mathrm{He}$ and $\mathrm{Na}$ are preferentially 
retained inside the cluster potential well, while $\mathrm{O}$ is mainly vented out through the galactic outflow. This naturally produces the Na-O anticorrelation and He-rich bMS population, as observed (Fig. 1, right-hand panels). The coexistence of populations with 'normal' and 'high' He content at $[\mathrm{Fe} / \mathrm{H}] \geqslant-1.4$ is expected if He is removed with different intensities from different regions of the proto-cluster.
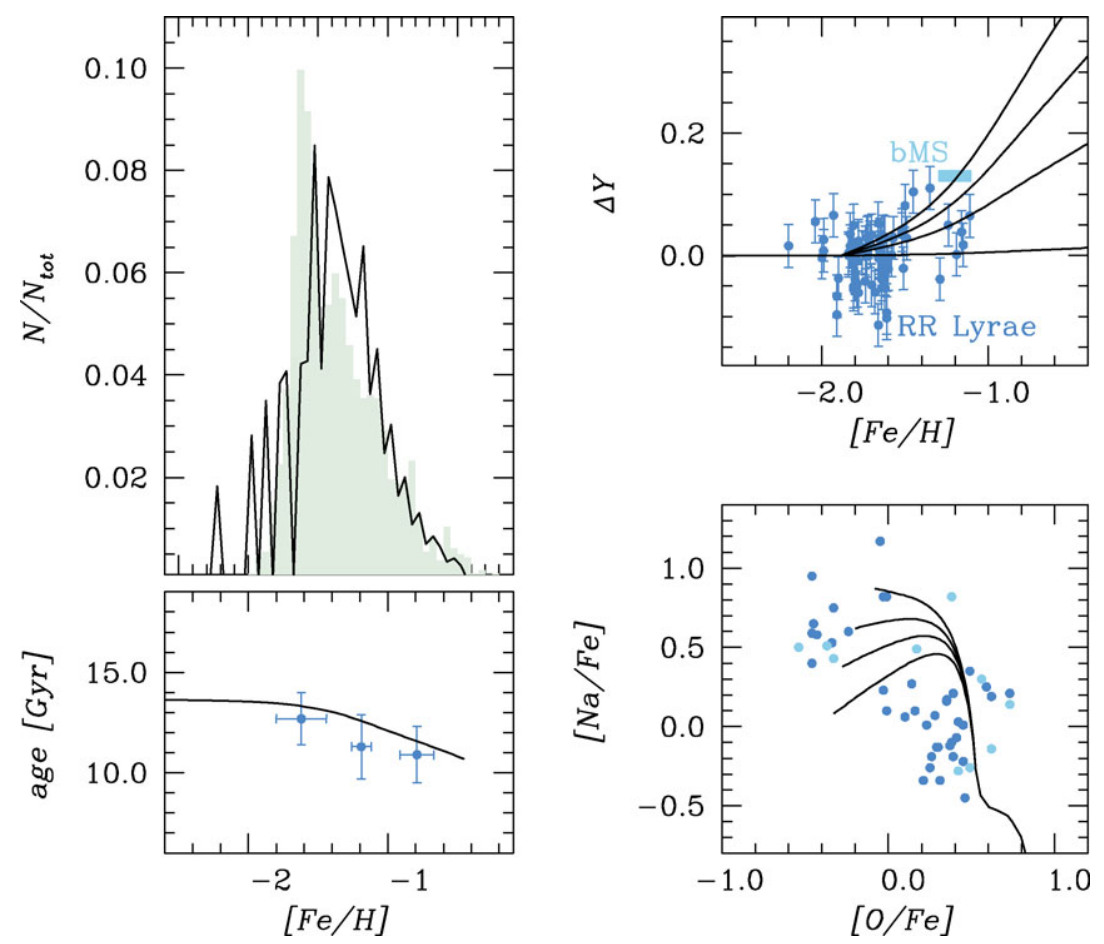

Figure 1. Predicted (thick solid lines) MDF (top left-hand panel), AMR (bottom left-hand panel), relative He enrichment (top right-hand panel) and Na-O anticorrelation (bottom righthand panel) for $\omega$ Cen stars compared to observations from Sollima et al. (2005; shaded histogram, top left-hand panel), Hilker et al. (2004; dots with error bars, bottom left-hand panel), Sollima et al. (2006; dots, top right-hand panel), Norris (2004; box, top right-hand panel), Norris \& Da Costa (1995) and Smith et al. (2000) (dots, bottom right-hand panel). The upper (lower) curves in the right-hand panels correspond to models computed with lower (higher) efficiencies of $\mathrm{He}$ and $\mathrm{Na}$ entrainment in the galactic outflow (see Romano et al. 2010).

\section{References}

Hilker, M., Kayser, A., Richtler, T., \& Willemsen, P. 2004, A\&A, 422, L9

Norris, J. E. 2004, ApJ, 612, L25

Norris, J. E. \& Da Costa, G. S. 1995, ApJ, 447, 680

Romano, D., Matteucci, F., Tosi, M., Pancino, E., Bellazzini, M., Ferraro, F.R., Limongi, M., \& Sollima, A. 2007, MNRAS, 376, 405

Romano, D., Tosi, M., Cignoni, M., Matteucci, F., Pancino, E., \& Bellazzini, M. 2010, MNRAS, in press (arXiv:0910.1299)

Smith, V. V., Suntzeff, N. B., Cunha, K., Gallino, R., Busso, M., Lambert, D. L., \& Straniero, O. 2000, AJ, 119, 1239

Sollima, A., Ferraro, F. R., Pancino, E., \& Bellazzini, M. 2005, MNRAS, 357, 265

Sollima, A., Borissova, J., Catelan, M., Smith, H. A., Minniti, D., Cacciari, C., \& Ferraro, F. R. 2006, ApJ, 640, L43 